Volume 11 Number 1 (February 2019) 48-54

\title{
Homogeneity of Mycoplasma agalctiae vaccine strains in an agalaxy- high-burden environment
}

\author{
Khatereh Kabiri ${ }^{1}$, Seyyed Ali Pourbakhsh ${ }^{2}$, Jamileh Norouzi ${ }^{1}$, Mohammad Sekhavati², \\ Keyvan Tadayon ${ }^{2 *}$
}

${ }^{1}$ Department of Microbiology, North Tehran Branch, Islamic Azad University, Tehran, Iran

${ }^{2}$ Agricultural Research, Education and Extension Organization (AREEO), Razi Vaccine and Serum Research Institute, Karaj, Iran

Received: October 2018, Accepted: January 2019

\begin{abstract}
Background and Objectives: Interrogation of the genomic relations between Iranian Mycoplasma agalactiae vaccine strains of Taliqan, Lorestan and Shiraz.

Materials and Methods: Two MLVA (covering VNTR loci of 5, 9, 17 and 19) and MLST (comprising $d n a A, g l t X, g y r B, C$, tufA genes) genotyping systems plus nucleotide structure analysis of P80 gene, was conducted.

Results: The shared MLVA pattern represented by the three strains differed to that of the Mag PG2 laboratory strain, only at locus VNTR19 where the PG2 genome hold a 3 bp longer stretch. In MLST analysis, at dnaA, gltX, gyrB, metS and tufA loci, the three strains displayed alleles 1,21, 2, 2 and 1, respectively. At, gltX locus a new allele (21) was detected where a new sequence type (ST33) was identified. Besides, the trio strains hold an identical nucleotide structure in their ma-mp81 gene. Conclusion: In explanation, lack of efficient disease control measures, has possibly contributed in evolution of a clone or a few clones that gradually overwhelmed the population over the time. Besides, the similarity between the Iranian and the PG2 strains, might be due to homoplasy or farming exercises such as animal importation. Inclusion of further local isolates in next studies will help to assess these assumptions.
\end{abstract}

Keywords: Mycoplasma agalactia; Vaccine; Strain; Homoplasy

"Corresponding author: Keyvan Tadayon, Ph.D, Agricultural Research, Education and Extension Organization (AREEO), Razi Vaccine and Serum Research Institute, Karaj, Iran.

Tel: +98-26-34502892

Fax: +98-26-34552194

Email: k.tadayon@rvsri.ac.ir

\section{INTRODUCTION}

Contagious agalactia (CA) is a highly infectious disease of small ruminants leading to extensive heavy losses to sheep and goat farming across the world. CA has been known for the last two centuries since its first description in Italy (1817) by Metaxa (1). 
It is reported from all five continents and is included in the List B of dangerous infections recognized by the International Office des Epizooties (OIE). In clinical view, CA behaves as a syndrome mainly affecting joins, mammary glands and eyes (2).

Four mycoplasma species are known to cause CA (3). These are Mycoplasma agalactiae (Mag), the world-known classic pathogen both in sheep and goats, Mycoplasma mycoides subsp. capri (Mmc), Mycoplasma capricolum subsp. capricolum (Mcc) and Mycoplasma putrefaciens (Mp) mainly in goats $(3,4)$.

CA actively infects sheep and goat populations in Mediterranean basin, Balkan region, Middle East, western Asia, and most of Africa (1).

In the Middle-Eastern Iran, Delpy and co-workers reported success in treatment of CA cases using Stovarsol sodique in early 1930s (5) but actually it was 1959 when the first description of CA clinical cases appeared in the literature (6). Since then numerous studies on microbiology, epidemiology, pathogenicity and control (7-10) of CA have been published by Iranian workers. A number of different vaccine preparations using exotic and indigenous strains of Mag have been employed to control the disease. While the national vaccination scheme includes annual application of as many as 20 million doses of agalaxy vaccine, Iran still experiences frequent epidemics of CA every year (8-11).

Current epidemiological knowledge of Mag has been recently broadened by application of molecular strategies such as variable number tandem repeats (VNTR), Multilocus sequence typing (MLST) and pulsed field gel electrophoresis (PFGE), Random amplified polymorphic DNA (RAPD) techniques (12). The level of genomic diversity of CA seems to be host-dependent as its populations in sheep are understood to be genetically less heterogenic (13) compared to that of the goats (14).

In the recent years VNTR typing systems have been employed in genetic studies of mycoplasmas including Mycoplasma hyorhinis (15), Mycoplasma hyopneumoniae (16), Mycoplasma bovis (17), Mycoplasma californicum (18) and Mycoplasma mycoides (19). Similarly, MLST genotyping has been used in epidemiological studies of several Mycoplasma species including M. agalactiae (20), Mycoplasma bovis (20). Mycoplasma hyopneumoniae (21), Mycoplasma hyorhinis (15) and Mycoplasma synoviae (22).

Molecular mass investigations of serum proteins from CA naturally infected sheep resulted in recognition a handful of immunodominant surface membrane proteins expressed in the early phase of infection $(23,24)$. One of these proteins, named P80 bearing a molecular mass of $80 \mathrm{kDa}$, is serum detectable in all sheep naturally infected by wild-type Mag isolates. The ma-mp81, the gene encoding P80 consists of 2, 166 nucleotides (24).

In 1940s, three local Mag isolates collected from Lorestan, Shiraz and Taliqan were selected for preparation of a bacterin vaccine. This liquid saponified biological has been ever manufactured and used in Iran with an average yearly vaccination rate of 20 million doses over the last decade. Anecdotal records show these three strains had differences in their biochemical properties at the time of isolation.

This study was intended to genetically characterize the Iranian trio Mag vaccine strains of Lorstan, Shiraz and Taliqan with MLST and MLVA methods. This genomic interrogation was further extended with analysis of their ma-mp81 gene compared to strains from rest of the world.

\section{MATERIALS AND METHODS}

Bacterial culture. Three Mag vaccine strains of Lorestan, Shiraz and Taliqan, preserved frozen in glass vials at $-70^{\circ} \mathrm{C}$ from the RVSRI bacterial archive, were revived through transfer of $5 \mathrm{ml}$ of the thawed material to two Falcon polyethylen tubes containing $45 \mathrm{ml}$ of PPLO broth supplemented with $15 \%$ sterile horse serum. Shaking incubation of the culture vessels $\left(37^{\circ} \mathrm{C}\right)$ continued for $72 \mathrm{~h}$ until expected turbidity attributed to the bacterial growth was achieved. Culture tubes were centrifuged $(3,000$ $\mathrm{g} / 5 \mathrm{~m}$ ) and deposition of each tube was transferred to a $1.5 \mathrm{ml}$ O-ring microfuge tube (containing $500 \mu \mathrm{l}$ TE buffer). Microtubes were submerged in a boiling water bath and heat-inactivated for $15 \mathrm{~m}$ when they were centrifuged again. The supernatant liquid, carrying the bacterial genomic material, was preserved in a fresh microtube and directly used for molecular experiments.

Confirmatory test. To authenticate identity of the trio strains, they were subjected to the specific PCR amplification based on the $u v r C$ gene using primer pair of MAGAUVRC1-L and MAGAUVRC1-R (25). 
PCR amplification. Analyses of VNTRs was conducted as previously described by McAuliffe et al. (12). Four VNTR loci of 5, 14, 17 and 19 were selected and targeted in the genetic assay.

The MLST analysis was performed based on five housekeeping genes using primer pairs and PCR amplification protocol developed by the McAulif et al. This scheme focuses on partial sequencing of $d n a A$, glt $X$, gyrB, metS and tufA genes.

For ma-mp81 gene structure analysis, four primer pairs were designed using the online software Primer 3 (https://www.ncbi.nlm.nih.gov/tools/primer-blast/) with the default settings and the Mag PG2 genome sequence (GenBank assembly accession: GCA_000063605.1, latest). In order to improve accuracy, designing and selection of primers were conducted in a way that flanking primer pairs shared a relatively large amount of target sequence as a total of 5,000 nucleotides were amplified and sequenced in search for the 2,166-bp long ma-mp81 gene (24).

Four VNTR analysis, PCR primer pairs were: VNTR 5, 5F (5'-AAA GAG AAA GGA AGC TGA A- 3') and 5R (5'-GGA TCA TTA TCG CTT TTT GA-3'); VNTR 14, 14F (5'-TTG AAA TAT CCG CTT AAG AAA-3') and 14R (5'-AAT TTG CAT TTA ATG GTG CT-3'); VNTR 17, 17F (5'-TTT AGC TTT TGA TTC AAT ACT TTC-3') and 17R (5'-AAA GAA TTA TGC GAG CAT TT-3'); and VNTR 19, 19F (5'-TTG CTT CTT GTG CTT CTT TT-3') and 19R (5'- AAG GGG ATC AAC CAG ATA AT-3').

For MLST analysis primers of PCR were $\operatorname{dnaA}, \mathrm{F}$ (5'-TAA CGT AAC CCC AAA CTC AC-3') and R (5'- CAT AAT TCA GGC GTC ATC TT-3'); gltX, F (5'- GCC TTG CCT ACA AAT CTT AT-3') and R (5'- ATA GTT GCT TAA GCG CAA AC-3') gyrB F (5'- CAA TAC ACA TCA ACC TTC CA-3') and R (5'- AGC AGA GTT ACC TTC GAC AA-3'); mets F (5'- GCC TGT AAA TTA GCC CTT CT-3') and R (5'-TTT TAA CCA AAA ATC AAG CTG-3'); tufA F (5'- GAA CAT GAT TAC TGG TGC TG-3') and R (5'- ACG GCC TTT TTC AAA TTC TA-3').

The PCR primers in ma-mp81 assay were $\mathrm{Mag}$ P80L F (5'- ATC AAA GGT GCT TGA GAA ATG G -3') and R (5'- ATA CTG GGG CAT TAG CAG ACA -3'); Mag P80M1 F (5'- GAG TAT GCT GAA GGT GAA AAC TCA -3') and R (5'- ACC CGC TGT TGA ACC TAT ACC A -3'); Mag P80M2 F (5'- AAA CTC AGC AGC ACA AAA CTC G -3') and R (5'GCG CCT CAA CTT ACA CCA AT -3'); Mag P80R F (5'- AAA TAA GGG CAC TGA AAT TGG TAC A
-3') and R (5'- GCT TCT TTA GCT TTA ATT GTG CCT -3').

PCR Amplifications were performed using an Eppendorf thermocycler in a final volume of $12 \mu \mathrm{l}$. Individual PCR reaction consists of $6 \mu$ of ready-to-use PCR master mix containing 2.5 units of Taq DNA polymerase, $200 \mathrm{mM}$ of each of the four dNTPs and $2 \mathrm{mM}$ MgSO4 (Ampliqon®, Denmark), $1 \mu \mathrm{l}$ of each forward and reverse primers $(5 \mathrm{pm} / \mu \mathrm{l}), 2.5 \mu \mathrm{l}$ of DNA template plus $1.5 \mu \mathrm{l}$ of double-distilled water to compensate the volume. In PCR-MLST reactions, an extra amount of $\mathrm{MgCl}_{2}(0.36 \mu \mathrm{l}$ from a $50 \mathrm{mM}$ solution) was used. The PCR cycling conditions were 45 sec at $95^{\circ} \mathrm{C}$, followed by 30 cycles of $45 \mathrm{sec}$ at $95^{\circ} \mathrm{C}$, $45 \mathrm{~s}$ at $56^{\circ} \mathrm{C}$ and $1 \mathrm{~min}$ at $72^{\circ} \mathrm{C}$, this was finished with a final extension step lasting for $10 \mathrm{~min}$ at $72^{\circ} \mathrm{C}$. Amplicons were kept at $4^{\circ} \mathrm{C}$ until analysis. Visualization of aliquots was conducted by electrophoresis on $1.5 \%$ agarose gel pre-stained with Red Safe ${ }^{\circledR}$ under UV illumination. The molecular weights were estimated using visual comparison of amplicons against a 100bp Molecular weight standard (26).

All the PCR amplicons from VNTR, MLST and ma-mp81 gene were sequenced using the same PCR primers at the sequencing facility of Macrogen, South Korea.

Sequencing chromatograms were visually checked and edited, if necessary, with Chromas lite (available at

http://www.technelysium.com.au/chromaslite.html).

AliView and MAFFT suites were consulted to determine the consensus sequence for each locus and also for assembling, trimming, concatenation and aligning the contigs. Tandem Repeat Finder (http://tandem.bu.edu/trf/trf.html) was employed in order to determine the number of repeats in each VNTR product.

VNTR profiles of strains were recorded as character data using allelic patterns. In MLST analysis, the assembled sequences of strains were subjected to the non-redundant Mycoplasma agalactiae MLST database (https://pubmlst.org/magalactiae/) to assign allele numbers and sequence types (STs).

To visualize the genetic relationship between $\mathrm{Mag}$ vaccine strains from Iran and the Mag population representing rest of the world in a single image, an analysis of VNTR findings was conducted based on a maximum-parsimony strategy. This was implemented using BioNumerics, version 6.7 where a matrix comprising 9 characters (5 MLST and 6 VNTR loci) was employed to encode the input data. 
To position the Iranian vaccine strains in the global picture of MLST profiles framed by the Mag MLST database, a Burst analysis was conducted using eBurst V3 (http://eburst.mlst.net).

\section{RESULTS}

In MLST analysis, the three Mag strains identically displayed alleles 1, 21, 2, 2 and 1 at dnaA, gltX, gyrB, metS and tufA loci, respectively. All but one of these alleles (the gltX allele, 21) were proved to be previously recorded according to the Mag MLST database. A new sequence type (ST33) with close similarity to ST4 was assigned to these strains (Fig. 1).
In MLVA analysis, all the three strains identically produced amplicons at VNTR5, VNTR14, VNTR17 and VNTR19 loci that were 626, 637, 533 and 594 bp in length, respectively. This resulted in obtaining an identical MLVA pattern for all the three strains. Compared to the Mag PG2 laboratory strain genome, a small difference was detected only at locus VNTR19 where the three Mag strains of Iran carried a 3 bp longer locus.

In analysis of the gene encoding P80 (ma-mp81), an identical substitution pattern of nucleotides between the three Iranian strains comparing to that of the Mag PG2 was recognized where at nucleotides 256, 446, 1411 and 2147, A, C, A and A in the genome of Mag PG2 substitutes with G, T, G and G in the three Iranians, respectively (Fig. 2).

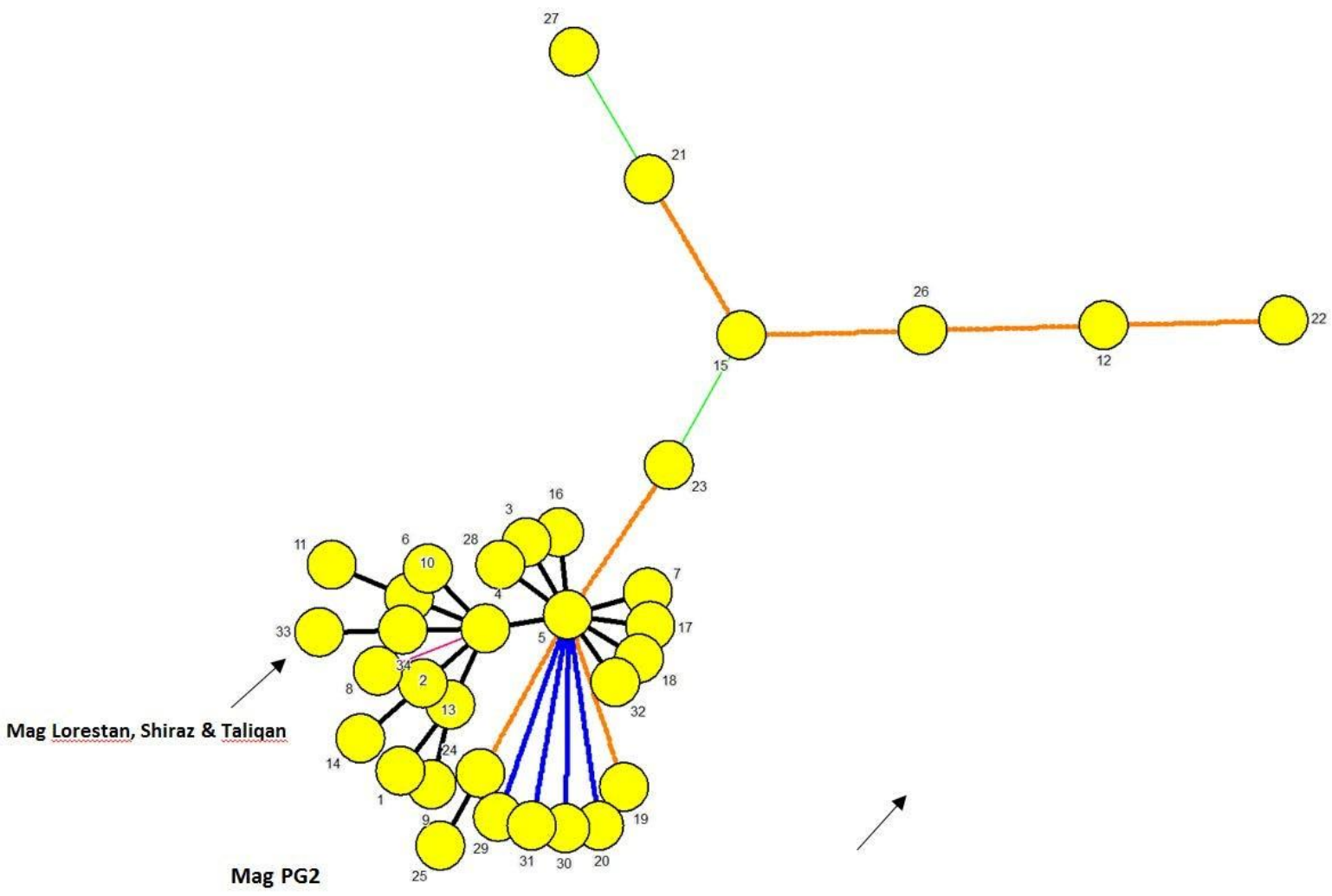

Fig. 1. Graphic representation of minimum spanning tree (MST) resulting from MLST typing of the 34 types registered with the international Mycoplasma agalactiae MLST Database (https://pubmlst.org/bigsdb?db=pubmlst_magalactiae_seqdef) as of January 2019. An identification policy based on recognition of all taxa with zero inter-taxon distance plus the priority rule using the Maximum number of $\mathrm{N}$-locus variants $(\mathrm{N}=1)$ were employed in generation of the MST. Here each MLST type is represented only once. MLST types differing by a single MLST locus are shown by thick short lines while longer thin connecting two MLVA types denote types while those with larger differing loci are denoted by thin longer dashed or dotted lines. The MLST type of the three Iranian and PG2 Ma vaccine and laboratory strains are marked. 


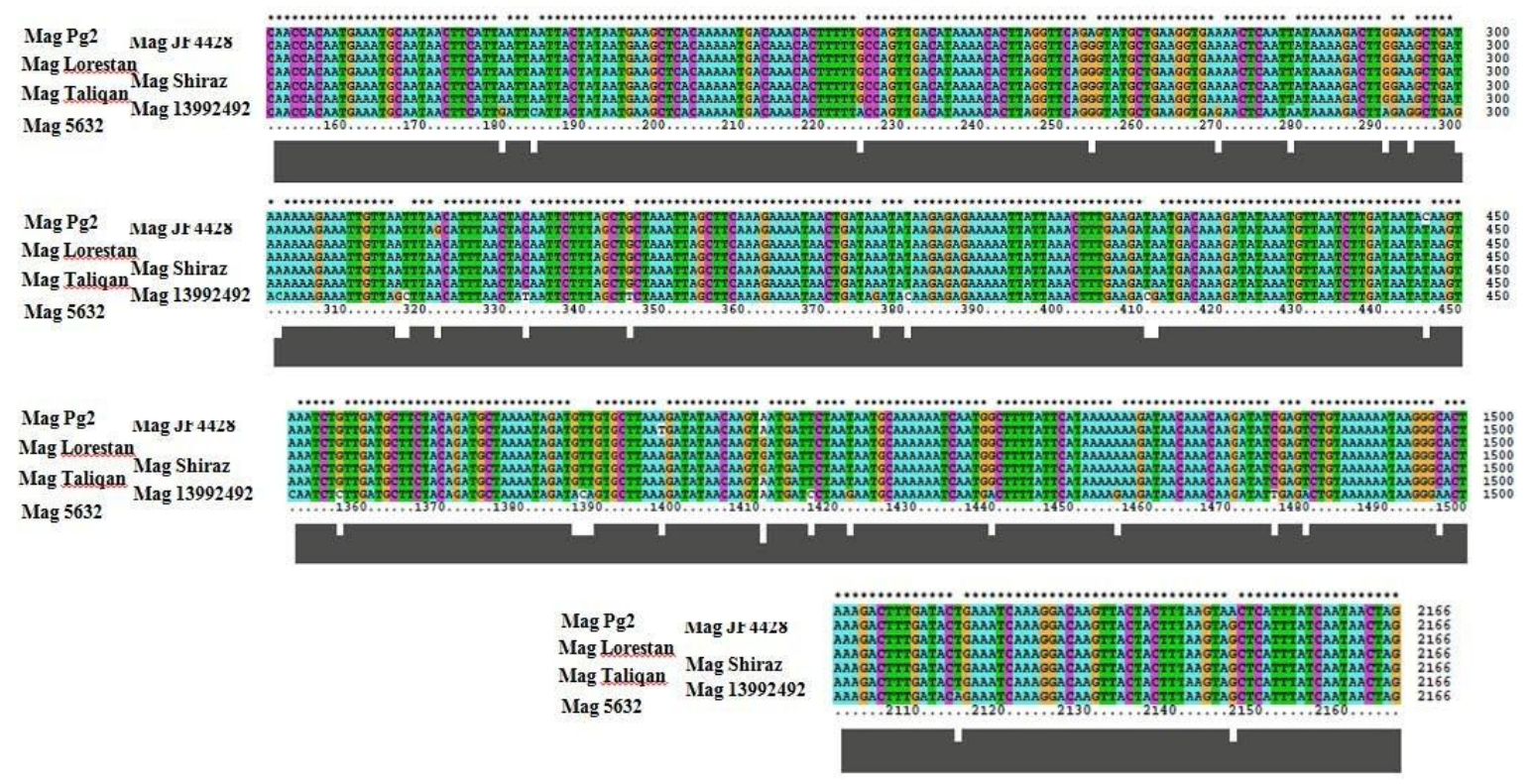

Fig. 2. Genetic diversity at ma-mp81 gene between the three Iranian vaccine and four laboratory strains of Mycoplasma agalctiae PG2, 5632, JF4428 and 13992492 as viewed by Clustal W. Asterisks depict identical matches of nucleotides. Numbers indicate nucleotide positions within the 2, 166 nucleotide-long ma-mp81 gene.

\section{DISCUSSION}

Contagious agalactiae is a poorly known disease of OIE-listed maladies where its socio-economic consequences are most felt by often poor farmers with small flocks of goats and sheep. The typically low profit margins of shepherding leads to limited interest from industry sector to fund researches aiming improve of conventional diagnosis and control measures against CA (27). This scenario happens in the Mediterranean basin and western Asia where farmers with few animals historically rear sheep and goat and continuous frequently-striking outbreaks of CA are reported. Iran along with Mongolia witness extensive burdens of CA in their farm animal populations.

The congruent observations made in this study by three approaches of VNTR, MLST and ma-mp81 gene analysis represented an identical genomic structure for the trio Mag strains of Lorestan, Shiraz and Taliqan.

Given the long history of ruminants farming in the region and the large distance between original isolation sites of these strains, the observed identical genetic pattern of them witnessed by MLST, MLVA and ma-mp81 gene structure, is unexpected. In explanation, one can hypothesize that lack of operating efficient disease control measures, has contributed in evolution of a highly successful clone or possibly a number of closely related few clones that existed in the region for some time with a rapid rise in their frequency and eventual overwhelming of the population over the time. In southern Europe this assumption has explained the population structure of Mag (28). The observed similarity between the Iranian and the PG2 strain shown by MLVA and MLST approaches on the other hand, is most likely to be due to be effect of the homoplasy phenomenon or animal husbandry exercises such as animal importation (Fig. 3). Inclusion of further local isolates in next studies will help to assess these assumptions. Given the characteristics of the Iranian animal farming sector, we assume circulation of more clonal complex or complexes of Mag in the Iranian environment is highly expectable. A further practical approach to improve our understanding from observations made by the present study is whole genome sequencing (WGS) of the trio Iranian strains to investigate possible differences among them. Considering the outcome from such study along with possibility for circulation of new clonal complexes of Mag not identified yet, a good question might be whether the current vaccine needs to be re-formulated. 


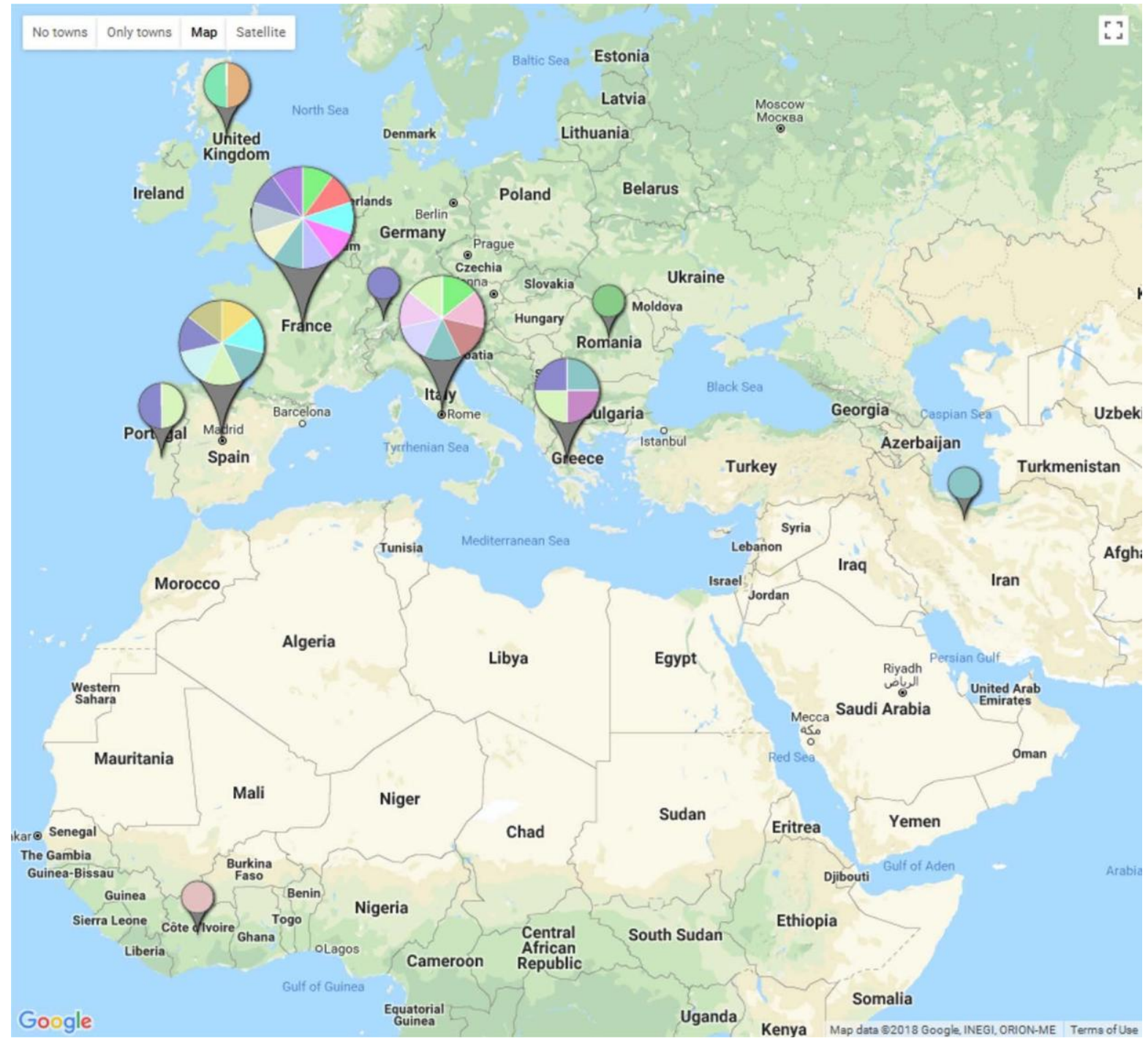

Fig. 3. Spatial distribution of 153 world Mycoplasma agalactiae isolates according to their MLVA allelic patterns. Different colors of pie charts reflect different MLVA types where larger pies represent more genotyped isolates. For details see the text.

\section{ACKNOWLEDGEMENTS}

The authors would like to acknowledge the financial support received from Razi Vaccine \& Serum Research Institute (RVSRI) to cover costs of this work. We wish to acknowledge the hard work and sincerity of our colleagues at the Veterinary Aerobic Bacteria Vaccine Department of RVSRI they provided in the course of this study.

\section{REFERENCES}

1. Madanat A, Zendulkova D, Pospíšil Z. Contagious agalactia of sheep and goats. A review. Acta Veterinaria 2001; 70: 403-412.

2. Bergonier D, Berthelot X, Poumarat F. Contagious agalactia of small ruminants: Current knowledge concerning epidemiology, diagnosis and control. Rev Sci Tech 1997; 16:848-873.

3. Olaogun OM, Kanci A, Barber SR, Tivendale KA, Markham PF, Marenda MS, et al. Survey of Victorian small ruminant herds for mycoplasmas associated with contagious agalactia and molecular characterisation of Mycoplasma mycoides subspecies capri isolates from one herd. Aust Vet J 2017; 95: 392-400.

4. Gomez-Martin A, Amores J, Paterna A, De la Fe C. 
Contagious agalactia due to Mycoplasma spp. In small dairy ruminants: Epidemiology and prospects for diagnosis and control. Vet J 2013; 198: 48-56.

5. Delpy LP (1938). Infectious diseases of farm animals in Iran 1st. Razi Institute. Iran.

6. Bory G, Entessar F. Etude analytique de l'immiunoprophylaxie de l'agalaxie contagieuse des chevres et des moutons. Arch Inst Razi 1959; 11: 48-52.

7. Baharsefat M, Manhouri H, Yamini B. Chemical composition of M. agalactiae. Arch Inst Razi 1967; 19: 8790.

8. Mohkber Dezfouli MR, Sadeghian S, Javanbakht J, Lakzian A. A study of occurrence and histopathology of mycoplasma infection in sheep in Tehran suburb, Iran. J Infect Dis Immun 2011; 3: 106-111.

9. Noaman V. Identification of Mycoplasma agalactiae by conventional and molecular methods on small ruminants in central zone of iran. Comp Clin Pathol 2015; 24: 653-657.

10. Heidari S, Derakhshandeh A, Firouzi R, Ansari-Lari M, Masoudian M, Eraghi V. Molecular detection of Chlamydophila abortus, Coxiella burnetii, and Mycoplasma agalactiae in small ruminants' aborted fetuses in southern Iran. Trop Anim Health Prod 2018; 50: 779785.

11. Pooladgar A, Looni R, Ghaemmaghami S, Pourbakhsh A, Ashtari A, Shirudi A. Isolation and identification of Mycoplasma agalactiae by culture and polymerase chain reaction (PCR) from affected sheep to contagious agalactia of Khuzestan province, Iran. Arch Inst Razi 2015; 70: 21-27.

12. McAuliffe L, Churchward CP, Lawes JR, Loria G, Ayling RD, Nicholas RA. Vntr analysis reveals unexpected genetic diversity within Mycoplasma agalacti$a e$, the main causative agent of contagious agalactia. BMC Microbiol 2008; 8: 193.

13. Nouvel LX, Marenda MS, Glew MD, Sagne E, Giammarinaro P, Tardy F, et al. Molecular typing of Mycoplasma agalactiae: Tracing European-wide genetic diversity and an endemic clonal population. Comp Immunol Microbiol Infect Dis 2012; 35: 487-496.

14. De la Fe C, Amores J, Tardy F, Sagne E, Nouvel LX, Citti C. Unexpected genetic diversity of Mycoplasma agalactiae caprine isolates from an endemic geographically restricted area of Spain. BMC Vet Res 2012; 8: 146.

15. Dos Santos LF, Clavijo MJ, Sreevatsan S, Rovira A, Moreira MA, Pieters M. Genotyping of Mycoplasma hyorhinis using multiple-locus variable number tandem repeat analysis. J Microbiol Methods 2015; 111:8792.

16. Pantoja LG, Pettit K, Dos Santos LF, Tubbs R, Pieters M. Mycoplasma hyopneumoniae genetic variability within a swine operation. $J$ Vet Diagn Invest 2016; 28 : 175-179.

17. Pinho L, Thompson G, Rosenbusch R, Carvalheira J. Genotyping of Mycoplasma bovis isolates using multiple-locus variable-number tandem-repeat analysis. $J$ Microbiol Methods 2012; 88: 377-385.

18. Hata E, Suzuki K, Hanyu H, Itoh M, Higuchi H, Kobayashi H. Molecular epidemiology of cases of Mycoplasma californicum infection in Japan. Appl Environ Microbiol 2014; 80: 7717-7724.

19. Nwankpa ND, Manso-silvan L, Lorenzon S, Yaya A, Lombin LH, Thiaucourt F. Variable number tandem repeat (VNTR) analysis reveals genetic diversity within Mycoplasma mycoides mycoides small colony isolates from nigeria. Vet Microbiol 2010; 146: 354-355.

20. Manso-Silván L, Dupuy V, Lysnyansky I, Ozdemir U, Thiaucourt F. Phylogeny and molecular typing of $M y$ coplasma agalactiae and Mycoplasma bovis by multilocus sequencing. Vet Microbiol 2012; 161: 104-112.

21. Mayor D, Jores J, Korczak BM, Kuhnert P. Multilocus sequence typing (mlst) of Mycoplasma hyopneumoni$a e$ : A diverse pathogen with limited clonality. Vet $\mathrm{Mi}$ crobiol 2008; 127: 63-72.

22. Harada K, Kijima-Tanaka M, Uchiyama M, Yamamoto T, Oishi K, Arao M, et al. Molecular typing of Japanese field isolates and live commercial vaccine strain of Mycoplasma synoviae using improved pulsed-field gel electrophoresis and vlha gene sequencing. Avian Dis 2009; 53: 538-543.

23. Tola S, Manunta D, Cocco M, Turrini F, Rocchigiani AM, Idini G, et al. Characterization of membrane surface proteins of Mycoplasma agalactiae during natural infection. FEMS Microbiol Lett 1997; 154: 355-362.

24. Tola S, Crobeddu S, Chessa G, Uzzau S, Idini G, Ibba $\mathrm{B}$, et al. Sequence, cloning, expression and characterisation of the 81-kda surface membrane protein (p80) of Mycoplasma agalactiae. FEMS Microbiol Lett 2001; 202: 45-50.

25. Subramaniam S, Bergonier D, Poumarat F, Capaul S, Schlatter Y, Nicolet J, et al. Species identification of Mycoplasma bovis and Mycoplasma agalactiae based on the uvrC genes by PCR. Mol Cell Probes 1998; 12: 161-169.

26. Sekhavati M, Tadayon K, Ghaderi R, Banihashemi R, Jabbari AR, Shokri G, et al. "In-house" production of DNA size marker from a vaccinal Bacillus anthracis strain. Iran J Microbiol 2015; 7: 45-49.

27. Loria GR, Nicholas RA. Contagious agalactia: The shepherd's nightmare. Vet J 2013; 198: 5-6.

28. Ariza-Miguel J, Rodriguez-Lazaro D, Hernandez M. Molecular characterization of Mycoplasma agalactiae reveals the presence of an endemic clone in Spain. $J$ Clin Microbiol 2013; 51: 656-660. 